\title{
Anti-inflammatory effects of a methanol extract of Dictamnus dasycarpus Turcz. root bark on imiquimod-induced psoriasis
}

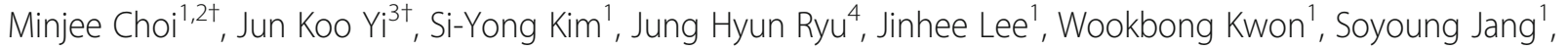
Dongjun Kim", MyoungOk Kim${ }^{5}$, Hyungwoo Kim, Sung Hyun Kim ${ }^{6}$, Seong-Kyoon Choi ${ }^{2^{*}}$ and Zae Young Ryoo ${ }^{1^{*}}$

\begin{abstract}
Background: The root bark of Dictamnus dasycarpus Turcz. has been successfully used for the treatment of inflammatory skin conditions such as eczema and pruritus. However, the anti-psoriatic effect of this plant has not until now been investigated.

Methods: The aim of this project was to investigate whether a methanol extract of Dictamnus dasycarpus Turcz. root bark (MEDD) can be used as a therapeutic agent for psoriasis in C57BL/6 mice model of imiquimod (IMQ)induced psoriasis. IMQ and MEDD was applied to mouse skin continuously for 7 days. The skin phenotype and the levels of inflammatory cytokines, such as interferon (IFN)- $y$ and interleukin (IL)-17, were analyzed. The immune cell population was determined by flow cytometry, and STAT1 and 3 protein levels were measured.

Results: An alleviation of scaly skin phenotype, immune cell infiltration in the dermis, and epidermal hyperplasia was observed after daily MEDD treatment in the lesion-affected area. It was also found that MEDD reduced IL-17 cytokine levels decreased by $44.37 \%(p<0.05)$, the number of IL-17-producing Th17 cells and $\gamma \delta T$ cells, and the size of the Th1 population secreting IFN- $\gamma$ decreased by $45.98,62.21$, and $44.42 \%$, respectively $(p<0.05)$, compared with the vehicle control group. STAT3 signals, associated with IL-17 are also reduced by MEDD.

Conclusions: An anti-psoriatic effect of MEDD was observed, as determined by decreased skin inflammation, reduced number of inflammatory cytokines, and a smaller population of inflammatory cells. These results contribute to the validation of the use of MEDD in the treatment of psoriasis.
\end{abstract}

Keywords: Dictamnus dasycarpus, Inflammation, Psoriasis, Skin, Gamma delta T cell, T helper cell

\section{Background}

Psoriasis is a chronic inflammatory skin disease characterized by epidermal keratinocyte hyper-proliferation caused by crosstalk between keratinocytes and immune cells [1], resulting in epidermal hyperplasia (acanthosis), retention of keratinocyte nuclei in the stratum corneum (parakeratosis), neutrophilic exudates in the epidermis (Munro's microabscess), and elongated rete ridges [2]. Many pro-inflammatory cytokines and chemokines,

\footnotetext{
* Correspondence: cskbest@dgist.ac.kr; jaewoong64@hanmail.net

${ }^{\dagger}$ Minjee Choi and JunKoo Yi contributed equally to this work

${ }^{2}$ Core Protein Resources Center, DGIST, Daegu, Republic of Korea

${ }^{1}$ School of Life Science, BK21 Plus KNU Creative Bioresearch Group,

Kyungpook National University, 80 Daehakro, Bukgu, Daegu 41566, South

Korea

Full list of author information is available at the end of the article
}

including tumor necrosis factor (TNF)- $\alpha$, interleukin (IL)-6, and IL-22, are associated with the aggravation of psoriatic lesions [3]. IL-17, which is produced mainly by $\mathrm{T}$ helper 17 (Th17) cells and $\gamma \delta \mathrm{T}$-cells, increases the proliferation of keratinocytes and augments keratinocyte gene expression involved in the immune response [4]. Increased levels of IL-17 in serum and at lesion sites have been reported in patients with psoriasis compared with those in healthy controls. Levels of this cytokine are correlated with disease severity [5]. T helper 1 (Th1) cells are the major producers of IFN- $\gamma$. IFN- $\gamma$ has been shown to be a potent promoter of Th17 cell function and trafficking of Th17 cells to immune sites, thereby exacerbating the pathogenesis of psoriasis [6].

(c) The Author(s). 2019 Open Access This article is distributed under the terms of the Creative Commons Attribution 4.0 International License (http://creativecommons.org/licenses/by/4.0/), which permits unrestricted use, distribution, and reproduction in any medium, provided you give appropriate credit to the original author(s) and the source, provide a link to the Creative Commons license, and indicate if changes were made. The Creative Commons Public Domain Dedication waiver (http://creativecommons.org/publicdomain/zero/1.0/) applies to the data made available in this article, unless otherwise stated. 
IL-17 and IFN- $\gamma$ are known to be regulated by the signal transducer STAT3 [7] and activator of transcription STAT1 [8] . Dysregulated STAT signaling is associated with chronic inflammatory disease and autoimmunity, and therefore STAT is a potential therapeutic target for this condition.

Dictamnus dasycarpus Turcz., a member of the family Rutaceae, is a perennial herbal plant widely distributed throughout Eastern Asia $[9,10]$. As a traditional remedy, the root bark of this plant has been used to treat skin diseases such as eczema, pruritus, urticaria, and atopic dermatitis [11]. The root bark of this plant contains many bio-active components, such as prenylated flavanone, limonoids, furoquinoline alkaloids, quinoline alkaloids, pyrrolidine alkaloids, sesquiterpenes, coumarins, and phenolic glycosides [12-19]. Previous studies have shown that extracts of this plant have anti-allergenic [20] and anti-inflammatory effects, including reduction of immune cell activity [21], proliferation [10], and recruitment [22]. However, the anti-inflammatory effects of this plant on psoriasis have not previously been studied.

The aim of this research was to investigate the effects of a methanol extract of Dictamnus dasycarpus Turcz., root bark (MEDD) in a mouse model of imiquimod (IMQ)-induced psoriasis. Therefore, the number of inflammatory cytokine immune cells was measured and the phenotype of the skin observed.

\section{Materials and methods \\ Chemicals and reagents}

Ethanol (Merck, Germany), acetone (Duksan, Korea), hair removal cream (Veet; Reckitt Benckiser, France), IMQ (Dong-a Otsuka, Korea), Vaseline (Unilever Korea, Korea), $70 \mu \mathrm{m}$ strainers (SPL life science, Korea), IL-17 ELISA kits (R\&D Systems MN, USA), and PerCP-Cy5.5Rat IgG1,k (RTK2071, Biolegend, USA) were used. First Strand cDNA Synthesis Kits and SYBR Premix EX Taq were purchased from Takara Bio (Japan).

CD16/CD32, fluorescein isothiocyanate (FITC)-antimouse CD4 (Mouse CTL clone V4) and FITC-Rat IgG2b,k (TNP-Keyhole Limpet Hemocyanin) were purchased from BD Biosciences (USA). FITC-anti-mouse $\gamma \delta$ TCR (UC7-13D5) and phycoerythrin (PE)-anti-mouse IL-17A, FITC-Armenian Hamster IgG (eBio299Arm), PE-anti-mouse IL-17A (eBio17B7), PerCP-Cy5.5-antimouse IFNY (XMG1.2), PE-anti-Rat IgG2a/k (eBR2a) and TRIzol reagent were purchased from Thermo Fisher Scientific (USA). Rabbit anti-STAT1(D1K9Y), rabbit anti-phospho-STAT1 (Tyr701), rabbit anti-STAT3 (79D7), rabbit anti-phospho-STAT3 (D3A6), rabbit antiSTAT5 (D2O6Y), rabbit anti-phospho-STAT5 (C11C5) and HRP-conjugated anti-rabbit IgG antibodies were purchased from Cell Signaling (USA). Mouse anti- $\beta$ -
Actin (C4) antibodies and HRP-conjugated m-IgGk BP were purchased from Santa Cruz Biotechnology (USA).

\section{Animals}

Because IMQ is known to cause more severe inflammation in female mice than in male mice [23] used sevento nine-week-old female wild-type C57BL/6 mice were used (Hyochang, Daegu, Korea). All animal experiments were performed according to the guidelines for animal experimentation and with permission from the Animal Use and Care Committee of Kyungpook National University (approval No.2018-0038). The mice were raised and maintained under conventional conditions in a room with a 12-h light/dark cycle, at a temperature of $23^{\circ} \mathrm{C}$, and were given free access to food and water. A minimum number of mice was used, to comply with the principles of replacement, refinement and reduction (the 3Rs).

\section{MEDD preparation}

The root bark of Dictamnus dasycarpus Turcz. was purchased from Kwang Myung Dang Medicinal Herbs (Ulsan, Korea). Before extraction, plant specimen was authenticated by professor Hyungwoo Kim, one of the authors of this article. MEDD (Voucher no.MH2010-010) was kindly provided by professor Hyungwoo Kim [11]. MEDD was deposited at the Division of Pharmacology, School of Korean Medicine, Pusan National University.

Before starting the experiment, $15 \mathrm{mg}$ MEDD was dissolved in $1 \mathrm{~mL}$ ethanol. The $1 \mathrm{ml}$ of ethanol with dissolved MEDD, and $1 \mathrm{ml}$ of ethanol alone as a vehicle control, were diluted with $4 \mathrm{ml}$ of acetone-olive oil (4:1) solution.

\section{Experimental design of IMQ-induced psoriasis and MEDD treatment}

Mice were anesthetized with Avertin (2.5\% tribromoethanol, intraperitoneally, $100 \mu \mathrm{L} / 10 \mathrm{~g}$ body weight). The dorsal skin of anesthetized mice was depilated using surgical scissors and hair removal cream. After depilation, mice were placed in individual cages. Mice were used in the experiment after resting for a day. The depilated mouse dorsal skin was treated daily for seven consecutive days with $62.5 \mathrm{mg}$ Aldara cream containing 5\% IMQ [24]. As a control for IMQ, the same volume of Vaseline was applied to the skin. After $6 \mathrm{~h}$ of IMQ treatment, $180 \mu \mathrm{g} / 60 \mu \mathrm{L}$ of MEDD or the same volume of vehicle control was applied daily to the depilated dorsal skin. The concentration of MEDD was treated at the same concentration that had previously had an antiallergic effect on mice skin in the previous experiment [11]. One day after the last treatment, the anesthetized mice were photographed for identification of the skin phenotype, and then euthanized by carbon dioxide inhalation. The home cage was filled with carbon dioxide through a regulator connected to a lid that fit on the top 
of the home cage, at a flow rate displacing $20 \%$ of the cage air per minute. The dorsal skin, draining lymph nodes and serum were collected from euthanized mice tissues for subsequent analysis. The body and spleen were weighed, as a measure of the severity of inflammation. A total of 20 mice were used. The mice used were divided into 4 groups and used as follows: 4 Vaseline, 5 IMQ, 4 IMQ + Vh(vehicle), 7 IMQ + MEDD. All experiments were performed in the laboratory.

\section{Histological analysis}

Mouse dorsal skin was fixed with $4 \%$ paraformaldehyde and embedded in paraffin. Tissue sections with a thickness of $5 \mu \mathrm{m}$ were stained with hematoxylin and eosin (H\&E). The thickness of the epidermis was measured based on the thin area between rete ridges, sebaceous glands, and hair follicles using Leica Application Suite software. The epidermis was measured four times per slide, for four slides per mouse. Using a grading scale of 0 to 4 , the severity of psoriasis was determined separately for three different areas: epidermal thickness, immune cell infiltration, and parakeratosis. Disease assessments were performed in a blinded manner by four researchers.

\section{Flow cytometry}

After isolation of draining lymph nodes, each was passed through a $70 \mu \mathrm{m}$ strainer to obtain single cells. Red blood cells were removed, and the cells were fixed with 4\% paraformaldehyde for $10 \mathrm{~min}$. Permeabilization was carried out on ice for $30 \mathrm{~min}$ using $90 \%$ methanol. To block Fc receptors, cells were reacted with anti CD16/ CD32 for 5 min before staining. FITC-anti-mouse CD4, PE-anti-mouse IL-17A, PerCP-Cy5.5-anti-mouse IFN $\gamma$, FITC-Rat IgG2b, $\mathrm{k}$, PE-anti-Rat IgG2a/k, and PerCPCy5.5-Rat IgG1, $\mathrm{K}$ antibodies were used to identify the populations of Th1 and Th17 cells. FITC-anti-mouse $\gamma \delta$ TCR and PE-anti-mouse IL-17A, FITC-Armenian Hamster IgG were used to analyze the population of $\gamma \delta \mathrm{T}$ cells producing IL-17. The cells were analyzed using a FACSAria III flow cytometer (BD Biosciences).

\section{Quantitative real time PCR}

Total RNA was extracted from skin lysates using TRIzol reagent, according to the manufacturer's protocol. The cDNA from total RNAs was synthesized using First Strand cDNA Synthesis Kits and measured using quantitative PCR with SYBR Premix EX Taq. To investigate the levels of mRNA for inflammatory cytokines (Tnf- $\alpha$, Ifng, Il6, Il17a, Il22, and $C c l 2$ ), real-time PCR was performed using a Step One Plus PCR system (Applied Biosystems, CA, USA). The expression levels of the genes were normalized to the expression of the Gapdh gene in each sample.

\section{Elisa}

IL-17 concentration in serum was measured using mouse IL-17 ELISA kit, according to the manufacturer's protocols.

\section{Western blot analysis}

The protein levels in the skin lysates were measured. Rabbit anti-STAT1, rabbit anti-phospho-STAT1, rabbit anti-STAT3, rabbit anti-phospho-STAT3, rabbit antiSTAT5, rabbit anti-phospho-STAT5 followed by HRPconjugated anti-rabbit IgG antibodies were used. Mouse anti- $\beta$-Actin antibodies, followed by HRP-conjugated anti-mouse IgGK BP antibodies were also used. Immunoblots were visualized using the ECL Detection System (GE Healthcare, Chicago, Illinois).

\section{Statistical analysis}

The results are expressed as median \pm interquartile range from at least three independent experiments. The significance of the differences between groups was calculated using Student's $t$-test. In all analyses, a $p$ value of less than 0.05 was considered to be statistically significant.

\section{Results \\ Effects of MEDD on symptom severity in IMQ-induced psoriasis model mice}

To investigate the effect of MEDD on psoriasis, IMQ followed by MEDD or a vehicle control were applied to the dorsal skin. After 7 days of application, erythema and silver-whitish scales were noted in the IMQ- and vehicle-treated groups; the symptoms in the MEDDtreated group were alleviated (Fig. 1a).

Enlargement of the spleen is an index of the severity of inflammation [25], so the ratio of spleen weight to body weight was calculated for each group. The spleens of the IMQ-treated groups were significantly enlarged compared with those of the Vaseline controls. However, MEDD treatment significantly decreased spleen weight compared to the vehicle controls (Fig. 1b). To observe the symptoms histologically, H\&E staining of the dorsal skin was performed 1 day after the last treatment. The IMQ and vehicle-treated groups showed thickened epidermis with elongated rete ridges (Fig. 1c arrow), parakeratosis (Fig. 1c arrowhead), and Munro's abscess (Fig. 1c asterisk). These symptoms were alleviated in the MEDD-treated group (Fig. 1c).

The thickness of the epidermis was measured, as an indicator of the severity of the psoriasis. Epidermal thickness significantly increased after IMQ treatment compared with Vaseline controls, whereas MEDD significantly decreased epidermal thickness relative to vehicle controls (Fig. 1d).

Histopathological severity was quantified via scoring of epidermal thickness, immune cell infiltration in the 
A
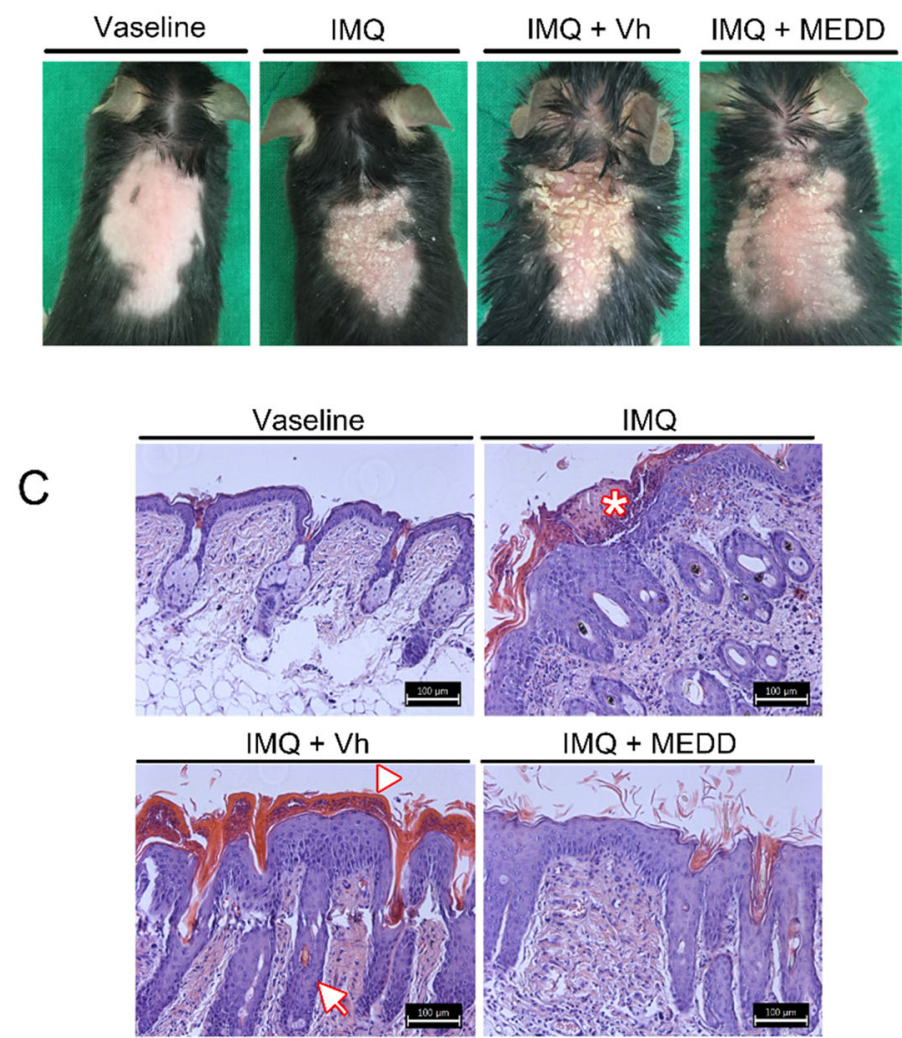

B
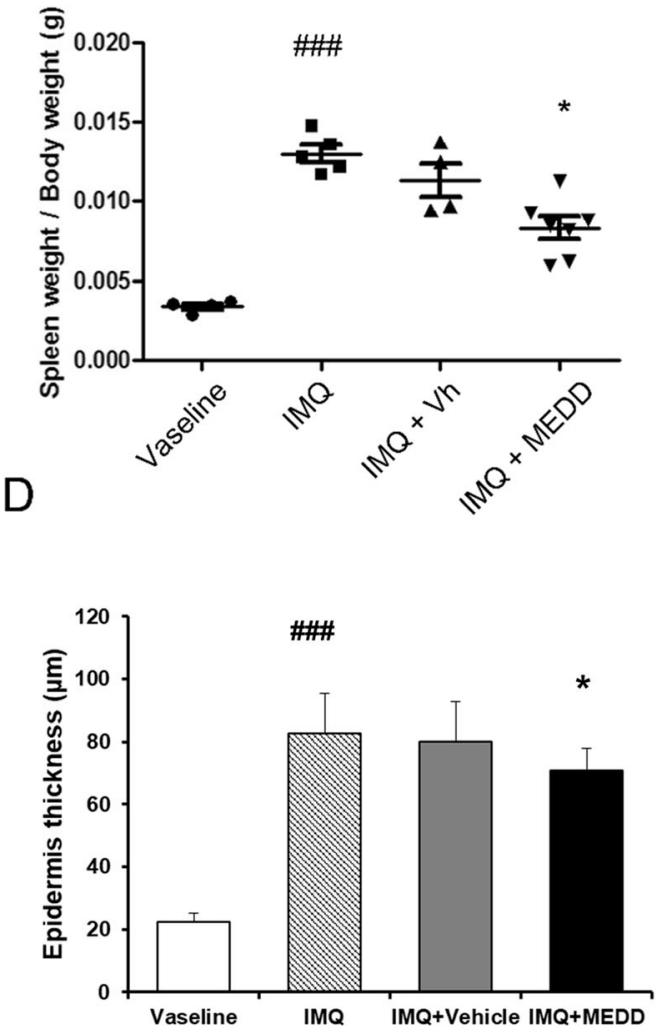

E

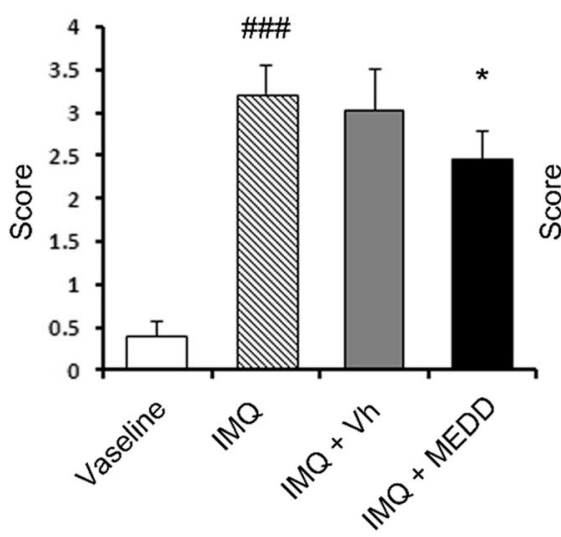

Cells infiltration

\#\#

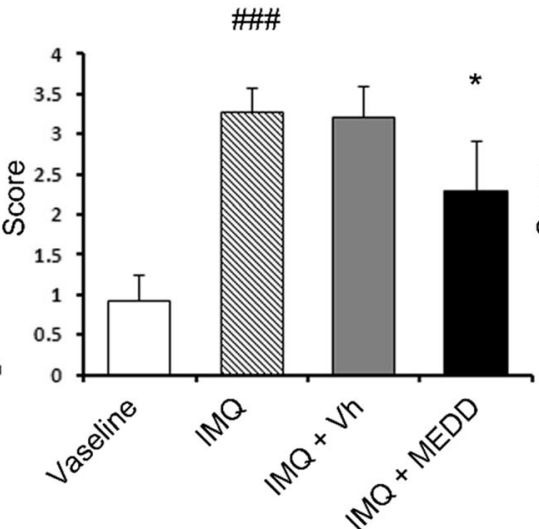

Parakeratosis

\#\#\#

Fig. 1 Effects of MEDD on symptom severity in IMQ-induced psoriasis model mice. The dorsal skin of mice was treated with Vaseline, imiquimod (IMQ), IMQ and Vh or MEDD for 7 days, and the phenotype was observed the next day. a Representative macroscopic views of the dorsal skin of each group of mice. $\mathbf{b}$ Calculation of spleen weight per body weight for each treatment group. Each dot represents one mouse. c Hematoxylin and eosin staining of paraffin sections from the dorsal skin of each group of mice. Note: Munro's abscess (asterisk), parakeratosis (arrowhead), and rete ridge (arrow). Scale bar: $100 \mu \mathrm{m}$ d Microscopic measurement of epidermal thickness $(\mu \mathrm{m}$ ) of dorsal skin. e Individual histological scoring of thickness, immune cell infiltration, and parakeratosis in the skin. $\mathbf{b}$, $\mathbf{d}$, e These results represent mean \pm S.D. of four independent experiments. $\mathbf{a}$, $\mathbf{c}$ The photograph is representative of four independent experiments. Statistical analysis was performed using Student's $t$-test. ${ }^{*} p<0.05$; ${ }^{* *} p<0.01$, compared with mice in the IMQ- and vehicle-treated group; \#\#\#, $p<0.001$, compared with Vaseline control mice; IMQ, imiquimod; Vh, Vehicle; MEDD, methanol extract of Dictamnus dasycarpus root bark. 
dermis area, and degree of parakeratosis. The increased severity of inflammation in the IMQ and vehicle control groups was significantly decreased by the application of MEDD (Fig. 1e). MEDD did appear to alleviate IMQinduced psoriasis-like symptoms.

\section{Effects of MEDD on inflammatory cytokine expression in IMQ-induced psoriasis}

To investigate the level of pro-inflammatory cytokines and chemokines in psoriatic dorsal skin lesions, realtime PCR was performed. IMQ treatment increased the level of mRNA for pro-inflammatory cytokines and chemokines, Tnf- $\alpha, I l 6, I l 22$, and $C c l 2$, and significantly increased Ifng and Ill7a compared to Vaseline controls. MEDD treatment decreased these pro-inflammatory cytokines, especially $I l 17 a$, compared with the vehicle controls $(p<0.05$, Fig. 2a).

IL-17 levels in the serum were found to be increased by IMQ compared with the Vaseline controls. MEDD treatment significantly decreased cytokine expression compared with vehicle controls (Fig. 2b). These results indicate that MEDD reduced pro-inflammatory cytokines, especially IL-17 level in the skin and also in the whole body.

\section{Effect of MEDD on immune cell population in IMQ-} induced psoriasis

Flow cytometry was used to investigate whether MEDD affects the populations of immune cells, the populations of $\mathrm{CD}^{+}{ }^{+} \mathrm{IFN}^{+}{ }^{+} \mathrm{T}$ helper 1 (Th1) cells and $\mathrm{CD} 4^{+} \mathrm{IL}-17 \mathrm{~A}^{+}$
T helper 17 (Th17) cells were increased by IMQ compared to the Vaseline controls and were significantly reduced by MEDD compared to the vehicle controls (Fig. 3a).

IL-17-producing $\gamma \delta \mathrm{T}$-cells are also associated with psoriasis [1]. Therefore, the population of $\mathrm{IL}-17^{+}$ $\gamma \delta \mathrm{TCR}^{+}$cells was measured. As in the Th17 cell population, the $\gamma \delta \mathrm{T}$ cell population was significantly decreased by MEDD compared to the vehicle controls (Fig. 3b).

It can therefore be concluded that MEDD reduced the population of Th1, Th17, and IL-17 producing $\gamma \delta \mathrm{T}$-cells.

\section{MEDD effects on STAT1 and STAT3 in IMQ-induced psoriasis}

STAT1 and STAT3 are the major factors that stimulate helper $\mathrm{T}$ cells to produce IFN $\gamma$ and IL-17, respectively [26]. The levels of these STAT proteins were investigated using Western blot analysis of skin lysates. The amount of phosphorylated STAT3 and total levels of STAT3 increased in the IMQ-treated groups compared with Vaseline controls. After MEDD treatment, the levels of phosphorylated STAT3 and total amounts of STAT3 were decreased compared with vehicle controls. However, there was no significant decrease in the amount of STAT1 by MEDD treatment (Fig. 4). Therefore, it can be concluded that MEDD reduced the phosphorylation and total amount of STAT3.

\section{Discussion}

This study produced evidence to indicate that there are anti-psoriasis effects of MEDD. Histological examination

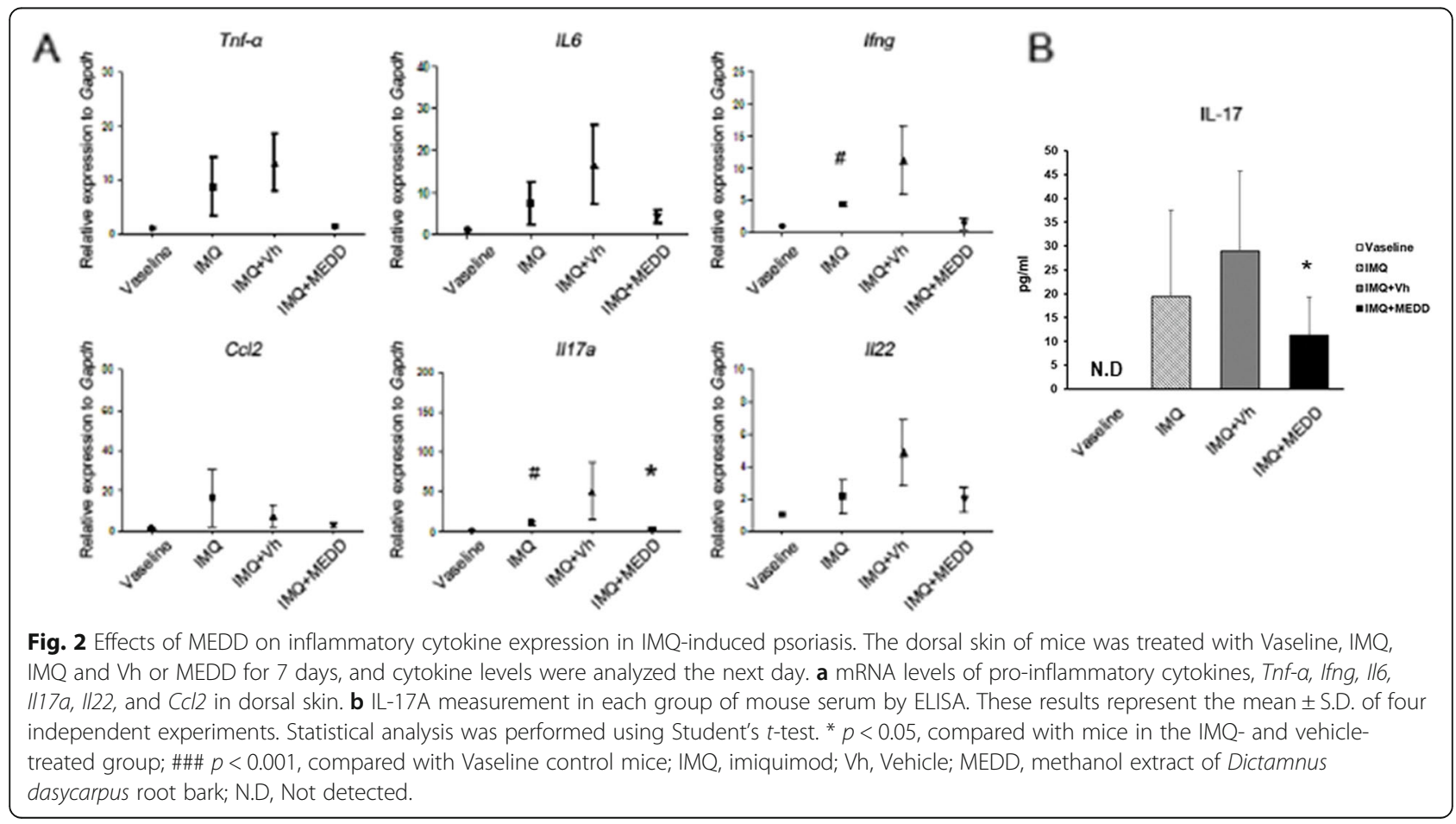




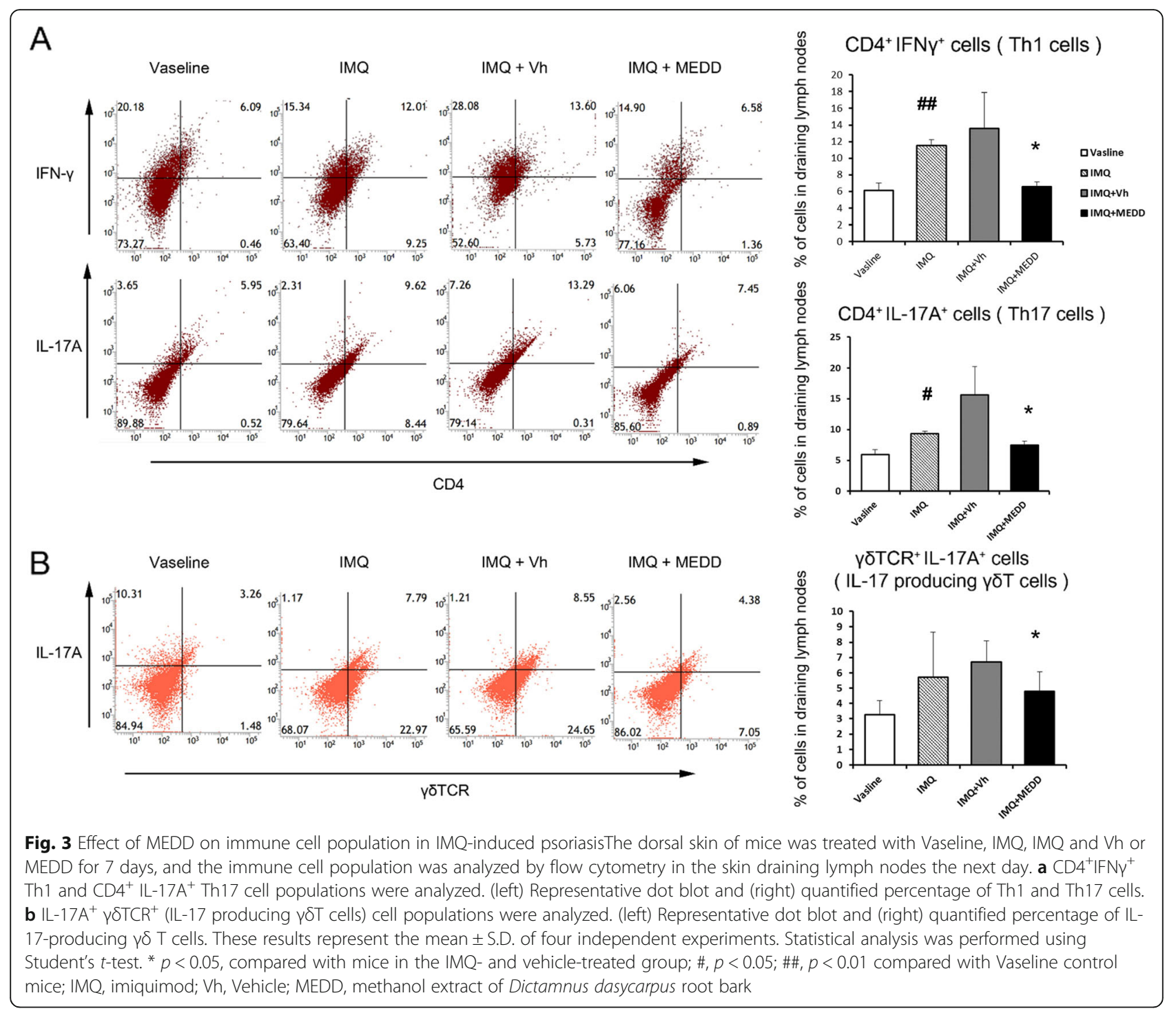

revealed that MEDD reduced the extent of scaly skin, decreased epidermal thickness, and alleviated parakeratosis. These symptoms are due to the hyper-proliferation of keratinocytes in psoriasis [3]. Therefore, it can be concluded that MEDD alleviated the hyperplasia of keratinocytes.

It was also evident that MEDD decreased the level of pro-inflammatory cytokines and the population of immune cells. Among the reduced inflammatory cytokines IL-17 was significantly reduced by MEDD compared with the vehicle controls. It was also apparent that MEDD reduced the numbers of IL-17-producing $\gamma \delta \mathrm{T}$ cells, Th17 cells, and IFN- $\gamma$-producing Th1 cells. Previous studies have shown that MEDD reduces IFN- $\gamma$ levels in allergic contact dermatitis [11]. However, this is the first study to show that MEDD downregulates IL-17 and decreases the population of immune cells producing IL-17.
MEDD reduced the total levels and phosphorylation of STAT3. Increased phosphorylation of STAT3 is known to stimulate the production of IL-17 [7]. Phosphorylation levels, as well as total amount, of these proteins is known to be associated with worsening of autoimmune diseases [27]. Therefore, decreased total protein levels of STAT3 is indicative of an anti-psoriasis effect of MEDD. Thus, it was demonstrated that MEDD inhibits IL-17 by decreasing STAT3. IL-17 is the major cause of deterioration in psoriasis. Therefore, it was concluded that MEDD relieved psoriasis symptoms by reducing the production of this cytokine.

The vehicle group had a more severe phenotype and greater inflammation than the IMQ-treated group. It is possible that the stress caused by the approximately 10 min of restraint needed to apply MEDD and vehicle to the lesion area on the mice increased inflammation [28]. In addition, repeated exposure of skin to acetone, a 


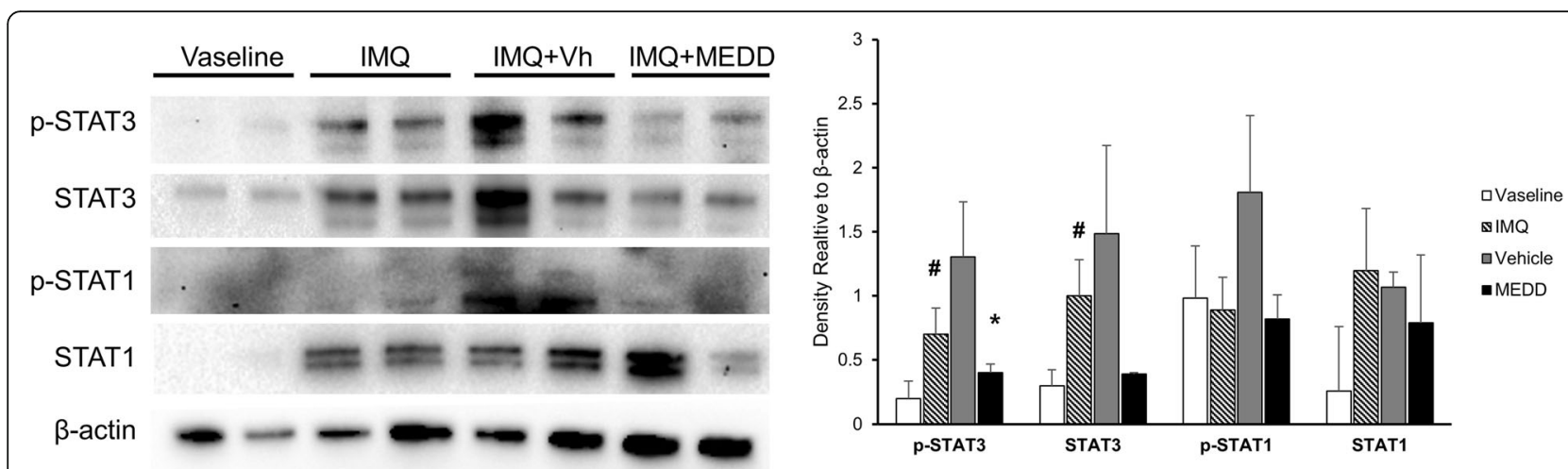

Fig. 4 MEDD effects on STAT1 and STAT3 in IMQ-induced psoriasisThe dorsal skin of mice was treated with Vaseline, IMQ, IMQ and Vh or MEDD for 7 days, and the immune cell population was analyzed by Western blotting in the skin lysates the next day. (left) Antibodies specific for PSTAT1, STAT1, PSTAT3, STAT3, and $\beta$-actin were used. Western blot performed on four samples per group. Among them, two samples per group were selected and are presented as representative images. (right) Densitometry quantification of proteins. Arrow indicates 86 (upper) and 79 (bottom) $\mathrm{kDa}$ size. These results represent mean \pm S.D. of four independent experiments. Statistical analysis was performed using Student's $t$-test. * $p<0.05$, compared with mice in the IMQ- and vehicle-treated groups; \#, $p<0.05$ compared with Vaseline control mice; IMQ, imiquimod; Vh, Vehicle; MEDD, methanol extract of Dictamnus dasycarpus root bark.

solvent in MEDD, may increase inflammation [29]. Despite these effects, the anti-inflammatory effect of MEDD on IMQ-induced psoriasis is apparent, because inflammation and phenotype are alleviated in the MEDD group compared to the vehicle group.

These anti-psoriatic effects of MEDD might be attributable to its bio-active components [12-19]. A previous study revealed that limonoids, quinoline alkaloids, and phenolic glycosides alleviate psoriasis symptoms [30-32]. The anti-psoriatic effects of MEDD may be caused by these bio-active components. The root bark of Dictamnus dasycarpus Turcz. has already been used extensively in East Asia [11]. Therefore, the anti-psoriasis effects observed in mice is expected to be transferrable to humans.

Also significant differences were obtained in this experiment even though a minimum number of mice were used to comply with the Replacement, Purification and Reduction Principle (3R).

Based on this study, it appears that MEDD has potential for use in the treatment of psoriasis.

\section{Conclusions}

In this study, MEDD reduced the numbers of inflammatory cells and IL-17 by decreasing STAT1 and STAT3 in IMQ-induced psoriasis. These results demonstrate the anti-psoriasis effects of MEDD. This study indicates that MEDD may be valuable as a remedy for psoriasis.

\section{Abbreviations}

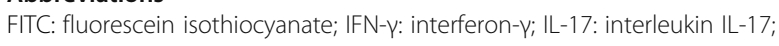
IMQ: imiquimod; MEDD: methanol extract of Dictamnus dasycarpus Turcz. root bark; PE: phycoerythrin; STAT: signal transducer and activator of transcription; Th1: T helper 1; Th17: T helper 17; TNF-a: tumor necrosis factor (TNF)- $a$

\section{Acknowledgements}

Not applicable.

\section{Authors' contributions}

The study was conceived and designed by MC and JY, ZYR. SC conducted the animal experiments and revised the manuscript. The experiments were conducted by SK. JHR, JL, WK, SJ, and DK analyzed the data. MOK and SHK contributed to study design, data analysis, and revising the manuscript. All authors read and approved the final manuscript.

\section{Funding}

This work was supported by the Korean Ministry of Science, Information and Communications Technology, and Future Planning (MSIP) National Research Foundation of Korea (NRF) (Grant 2016R1A2B4014686, 2017R1A2B4007888 and 2018R1D1A1B07041030) and by the Korean Government Ministry of Trade, Industry and Energy (N0001822). The funding bodies have no role in the design of the study and collection, analysis, and interpretation of data in writing the manuscript, which are fully the responsibilities of the authors.

\section{Availability of data and materials}

Not applicable.

The datasets used and/or analyzed during the current study available from the corresponding author on reasonable request.

\section{Ethics approval and consent to participate}

All animal experiments were performed according to the guidelines for animal experimentation and with permission from the Animal Use and Care Committee of Kyungpook National University (approval No.2018-0038).

\section{Consent for publication}

Not applicable.

\section{Competing interests}

The authors declare that they have no competing interests.

\section{Author details}

${ }^{1}$ School of Life Science, BK21 Plus KNU Creative Bioresearch Group, Kyungpook National University, 80 Daehakro, Bukgu, Daegu 41566, South Korea. ${ }^{2}$ Core Protein Resources Center, DGIST, Daegu, Republic of Korea. ${ }^{3}$ Gyeongsangbukdo Livestock Research institute, Yeongju, South Korea. ${ }^{4}$ Pusan National University School of Korean Medicine, Busan, South Korea. ${ }^{5}$ School of Animal Science Biotechnology, Kyungpook National University, Daegu, South Korea. ${ }^{6}$ Department of Bio-Medical Analysis, Korea Polytechnic College, Chungnam, South Korea. 
Received: 25 October 2018 Accepted: 21 November 2019

Published online: 02 December 2019

\section{References}

1. Chiricozzi A, Romanelli P, Volpe E, Borsellino G, Romanelli M. Scanning the immunopathogenesis of psoriasis. Int J Mol Sci. 2018:19(1).

2. Sabat R, Philipp S, Hoflich C, Kreutzer S, Wallace E, Asadullah K, Volk HD, Sterry W, Wolk K. Immunopathogenesis of psoriasis. Exp Dermatol. 2007; 16(10):779-98.

3. Albanesi C, Madonna S, Gisondi P, Girolomoni G. The interplay between keratinocytes and immune cells in the pathogenesis of psoriasis. Front Immunol. 2018;9:1549.

4. Malakouti M, Brown GE, Wang E, Koo J, Levin EC. The role of IL-17 in psoriasis. J Dermatol Treat. 2015;26(1):41-4.

5. Yilmaz SB, Cicek N, Coskun M, Yegin O, Alpsoy E. Serum and tissue levels of IL-17 in different clinical subtypes of psoriasis. Arch Dermatol Res. 2012; 304(6):465-9.

6. Kryczek I, Bruce AT, Gudjonsson JE, Johnston A, Aphale A, Vatan L, Szeliga W, Wang Y, Liu Y, Welling TH, et al. Induction of IL-17\&lt;sup\&gt; \&lt;/ sup\&gt; T cell trafficking and development by IFN- $Y$ : mechanism and pathological relevance in psoriasis. J Immunol. 2008;181(7):4733.

7. Annalisa Camporeale VP. IL-6, IL-17 and STAT3 a holy trinity in autoimmunity.Pdf. Front Biosci. 2012;17(6):2306-26.

8. Hu X, Ivashkiv LB. Cross-regulation of signaling pathways by interferongamma: implications for immune responses and autoimmune diseases. Immunity. 2009:31(4):539-50.

9. Kim C, Shin M, An D, Lee K: The encyclopedia of oriental herbal medicine 1997:1648-1651.

10. Choi SP, Choi CY, Park K, Kim N, Moon HS, Lee D, Chun T. Glabretal-type triterpenoid from the root bark of Dictamnus dasycarpus ameliorates collagen-induced arthritis by inhibiting Erk-dependent lymphocyte proliferation. J Ethnopharmacol. 2016;178:13-6.

11. Kim H, Kim M, Kim H, Lee GS, An WG, Cho SI. Anti-inflammatory activities of Dictamnus dasycarpus Turcz., root bark on allergic contact dermatitis induced by dinitrofluorobenzene in mice. J Ethnopharmacol. 2013;149(2): 471-7.

12. Chang J, Xuan L-J, Xu Y-M, Zhang J-S. Seven new sesquiterpene glycosides from the root bark of Dictamnus dasycarpus. J Nat Prod. 2001;64(7):935-8.

13. Chang J, Xuan L-J, Xu Y-M, Zhang J-S. Cytotoxic terpenoid and immunosuppressive phenolic glycosides from the root bark of Dictamnus dasycarpus. Planta Med. 2002:68(05):425-9.

14. Guo L-N, Pei Y-H, Chen G, Cong H, Liu J-C. Two new compounds from Dictamnus dasycarpus. J Asian Nat Prod Res. 2012;14(2):105-10.

15. Jeong SH, Han XH, Hong SS, Hwang JS, Hwang JH, Lee D, Lee MK, Ro JS, Hwang BY. Monoamine oxidase inhibitory coumarins from the aerial parts of Dictamnus albus. Arch Pharm Res. 2006;29(12):1119-24.

16. Kang SS, Kim JS, Son KH, Chang HW, Kim HP. A new prenylated flavanone from the roots of Sophora flavescens. Fitoterapia. 2000;71(5):511-5.

17. K-W N, Je K-H, Shin Y-J, Kang SS, Mar W. Inhibitory effects of furoquinoline alkaloids fromMelicope confusa andDictamnus albus against human phosphodiesterase 5 (hPDE5A)in vitro. Arch Pharm Res. 2005;28(6):675-9.

18. Souleles C. Flavonoids from Dictamnus albus. Planta Med. 1989:55(4):402

19. Yang J-L, Liu L-L, Shi Y-P. Limonoids and Quinoline alkaloids from Dictamnus dasycarpus. Planta Med. 2011;77(03):271-6.

20. Jiang S, Nakano Y, Rahman MA, Yatsuzuka R, Kamei C. Effects of a Dictamnus dasycarpus T. extract on allergic models in mice. Biosci Biotechnol Biochem. 2008;72(3):660-5.

21. Ghosh C, Hong B, Batabyal S, Jeon TI, Yang SH, Hwang SG. Antiinflammatory activity of the ethanol extract of Dictamnus dasycarpus leaf in lipopolysaccharide-activated macrophages. BMC Complement Altern Med. 2014;14(330):330

22. Han HY, Ryu MH, Lee G, Cheon WJ, Lee C, An WG, Kim H, Cho SI. Effects of Dictamnus dasycarpus Turcz., root bark on ICAM-1 expression and chemokine productions in vivo and vitro study. J Ethnopharmacol. 2015; 159:245-52.

23. Alvarez $P$, Jensen LE. Imiquimod treatment causes systemic disease in mice resembling generalized Pustular psoriasis in an IL-1 and IL-36 dependent manner. Mediat Inflamm. 2016;2016:6756138.

24. van der Fits L, Mourits S, Voerman JS, Kant M, Boon L, Laman JD, Cornelissen F, Mus AM, Florencia E, Prens EP, et al. Imiquimod-induced psoriasis-like skin inflammation in mice is mediated via the IL-23/IL-17 axis. J Immunol. 2009:182(9):5836-45.

25. Brown NF, Marks DJ, Smith PJ, Bloom SL. Splenomegaly. British Journal of Hospital Medicine (London, England : 2005). 2011;72(11):M166-9.

26. Christie D, Zhu J. Transcriptional regulatory networks for CD4 T cell differentiation. Curr Top Microbiol Immunol. 2014;381:125-72.

27. Sano S, Chan KS, Carbajal S, Clifford J, Peavey M, Kiguchi K, Itami S, Nickoloff BJ, DiGiovanni J. Stat3 links activated keratinocytes and immunocytes required for development of psoriasis in a novel transgenic mouse model. Nat Med. 2005;11(1):43-9.

28. Voorhees JL, Tarr AJ, Wohleb ES, Godbout JP, Mo X, Sheridan JF, Eubank TD, Marsh CB. Prolonged restraint stress increases $I L-6$, reduces $I L-10$, and causes persistent depressive-like behavior that is reversed by recombinant IL-10. PLoS One. 2013;8(3):e58488.

29. Evans FJ, Schmidt RJ. An assay procedure for the comparative irritancy testing of esters in the tigliane and daphnane series. Inflammation. 1979; 3(3):215-23.

30. Parlapally S, Cherukupalli N, Bhumireddy SR, Sripadi P, Anisetti R, Giri CC, Khareedu VR, Reddy Vudem D. Chemical profiling and anti-psoriatic activity of methanolic extract of Andrographis nallamalayana J.L.Ellis. Nat Prod Res. 2016;30(11):1256-61.

31. Muhammed Majeed PD, Satyan, K.S., Lakshmi Prakash: Neem oil limonoids Product overview. 2007.

32. Foreman Ml, Taylor M, Clark C, Devitt H, Hanlon G, Kelly I, Lukowiecki G. Pharmacology and treatment Isoquinoline is a possible anti-psoriatic agent in coal tar. Br J Dermatol. 1985;112(3):323-8.

\section{Publisher's Note}

Springer Nature remains neutral with regard to jurisdictional claims in published maps and institutional affiliations.

Ready to submit your research? Choose BMC and benefit from:

- fast, convenient online submission

- thorough peer review by experienced researchers in your field

- rapid publication on acceptance

- support for research data, including large and complex data types

- gold Open Access which fosters wider collaboration and increased citations

- maximum visibility for your research: over $100 \mathrm{M}$ website views per year

At $\mathrm{BMC}$, research is always in progress.

Learn more biomedcentral.com/submissions 